Eur. J. Clin. Chem. Clin. Biochem.

Vol. 31, 1993, pp. $459-465$

(C) 1993 Walter de Gruyter \& Co.

Berlin - New York

\title{
Galactosyl Hydroxylysine and Deoxypyridinoline: A Methodological Comparison ${ }^{1}$ )
}

\author{
By P. Bettica ${ }^{1}$, D. J. Baylink ${ }^{2}$ and L. Moro ${ }^{1}$ \\ 1 Dipartimento di Biochimica, Biofisica e Chimica delle Macromolecole, Universita' degli Studi di Trieste, \\ Trieste, Italy \\ 2 Department of Mineral Metabolism, Jerry L. Pettis Veterans Hospital, Loma Linda, California, USA
}

(Received January 1/April 6, 1993)

\begin{abstract}
Summary: Galactosyl hydroxylysine and deoxypyridinoline are at present the most promising markers of bone resorption. Various studies have indeed shown that these two markers discriminate with high accuracy subjects with different rates of bone turnover and that their accuracies and discriminate power are very similar. The aim of this paper is to compare the practicality and the reproducibility of the HPLC galactosyl hydroxylysine and deoxypyridinoline assays.
\end{abstract}

In summary, this review shows that the galactosyl hydroxylysine and deoxypyridinoline HPLC assays differ mainly in the need, in using deoxypyridinoline, for an acid hydrolysis and a preextraction of the urine samples. This implies two major problems for deoxypyridinoline: 1) more time is required due to the cumbersome preanalytical procedures; and 2) a lower reproducibility. Our data, in fact, show that both the intra-assay and inter-assay coefficient of variation of the deoxypyridinoline assay are almost $100 \%$ higher than those of the galactosyl hydroxylysine assay.

\section{Introduction}

Because of the importance of bone resorption in the pathogenesis of bone diseases, there is an increasing demand for non-invasive techniques for the rapid and easy detection of changes in the rate of this process. Biochemical markers are certainly adequate for this purpose, and the last decade has seen the development of new markers that appear to be specific for bone resorption. Because type 1 collagen is the most abundant protein of the bone matrix, most of the assays for estimating bone resorption rate measure the urinary excretion of different products of collagen breakdown (hydroxyproline, galactosyl hydroxylysine and deoxypyridinoline).

This review of the present knowledge of galactosyl hydroxylysine and deoxypyridinoline also compares

\footnotetext{
') Funding organization: Italian Ministry of the University and Scientific Technol. Res.-MURST
}

the techniques used to measure these two markers with particular emphasis on their practicality and their reproducibility.

\section{Rationale for the Use of Galactosyl Hydroxylysine and Deoxypyridinoline as Markers for Bone Resorption}

Type 1 collagen is the most abundant protein in the bone matrix. This unique protein, which accounts for almost $80 \%$ of the collagens present in the body and which, in addition to bone, is also present in other connective tissues, such as skin, is a heterotrimer formed by two $\alpha_{1}(\mathrm{I})$-chains and one $\alpha_{2}$-chain folded together in a triple helix. In bone this molecule is produced by the osteoblasts and deposited in a highly organized manner in the matrix (for a review on collagen synthesis see $1 . c .(1,2)$.

After the synthesis on the ribosomes, the collagen chains undergo different post-translational modifica- 
tions both inside and outside the cell. While no differences exist in the primary structure of the type 1 collagen deposited in different connective tissues, the post-translational modifications are, to different extents, tissue-specific (1).

After the pro- $\alpha$-chains have entered the endoplasmic cisternae, approximately 100 proline and ten lysine residues in every chain are hydroxylated by specific enzymes to hydroxyproline and hydroxylysine. Furthermore, either a galactose or a glucose-galactose is attached to approximately one third of the hydroxylysine residues by specific transferases, forming galactosyl hydroxylysine and glucosyl-galactosyl hydroxylysine. As soon as at least 90 proline residues are converted to hydroxyprolines, two pro- $\alpha_{1}(\mathrm{I})$ chains and one pro- $\alpha_{2}$-chain fold together, an event which inhibits any further transformation and results in the formation of type 1 procollagen.

The procollagen molecule is then excreted by the cell into the extracellular space where the two non-helical extensions, called $\mathrm{N}$ - and $\mathrm{C}$-terminal procollagen peptides, are cleaved off by two specific peptidases. The new collagen thus formed is then inserted in the existing matrix and some of the lysines are oxidatively deaminated by lysyl oxidase. Finally, the oxidized lysines form reducible and non-reducible crosslinks within and between the collagen molecules.

Because the products of these post-translational modifications show a certain tissue specificity, and are released and excreted in the urine during the collagen breakdown, they have been proposed as markers of bone resorption.

\section{Hydroxyproline: The Time-Honoured Marker of Bone Resorption}

Determination of the urinary excretion of hydroxyproline is the most commonly used routine clinical assay for the measurement of bone resorption.

However, hydroxyproline is not specific for bone collagen, since together with proline and alanine it is the most abundant amino acid (except for glycine) in all collagen molecules (3). In addition, hydroxyproline is present in all molecules that have a collagen-like structure, such as C1q component of complement (4).

Moreover, urinary hydroxyproline excretion cannot be used to quantify the rate of collagen breakdown, since about $80 \%$ of hydroxyproline released during this process is metabolized in the liver to $\mathrm{H}_{2} \mathrm{O}$ and $\mathrm{CO}_{2}(5,6)$, and a fraction of the hydroxyproline (perhaps $10 \%$ ) that is detectable in urine is derived from newly synthesized procollagen peptides (7) and new collagen which is degraded before being deposited in the matrix (8).

\section{Galactosyl Hydroxylysine and Deoxypyridinoline: Two New Specific Markers of Bone Resorption}

In recent years two different products of collagen breakdown have been proposed as specific markers of bone resorption. These molecules are products of intracellular (galactosyl hydroxylysine) and extracellular (deoxypyridinoline) post-translational collagen modifications.

Hydroxylysine glycosides are specific for collagen. Although the fraction of hydroxylysine residues that are glycosylated does not differ in skin or bone (these are the major sources of type 1 collagen in the human body) the pattern of hydroxylysine glycosylation is different in these two tissues. Thus glucosyl-galactosyl hydroxylysine predominates in the skin, where the ratio between glucosyl-galactosyl hydroxylysine and galactosyl hydroxylysine (glucosyl-galactosyl hydroxylysine/galactosyl hydroxylysine) is approximately 2 (9), whereas galactosyl hydroxylysine predominates in bone, where the glucosyl-galactosyl hydroxylysine/galactosyl hydroxylysine ratio is 0.47 (10).

This pattern of glycosylation, moreover, does not seem to vary in the presence of increased bone turnover, since the glucosyl-galactosyl hydroxylysine/galactosyl hydroxylysine ratio in bone collagen is not altered in Paget's disease of bone (10). Recently some concerns have been expressed on the possibility of a conversion of glucosyl-galactosyl hydroxylysine to galactosyl hydroxylysine by a kidney $\alpha$-glucosidase demonstrated in the rat (10b). However, Moro et al. have recently demonstrated that no $\alpha$ - or $\beta$-glycosidases exist in the human kidney, so that glucosylgalactosyl hydroxylysine and galactosyl hydroxylysine are indeed the final products of collagen degradation in humans (10c).

Finally the hydroxylysine glycosides do not undergo any major liver metabolism and their urinary excretion is not influenced by the diet $(10,11,11 \mathrm{~b})$.

The pyridinium crosslink, i.e. pyridinoline, also called hydroxylysyl-pyridinoline, and deoxypyridinoline, also named lysyl-pyridinoline, are the most important non-reducible crosslinks of mature bone collagen.

Pyridinoline is the most abundant of the pyridinium crosslinks and is present in different connective tissues, including bone, with the highest concentration in cartilage (12). Deoxypyridinoline, on the other hand, has been detected only in bone and dentine, 
where its concentration is approximately one fourth of that of pyridinoline (12).

Studies in animals have shown that the amount of pyridinoline and deoxypyridinoline in bone is not constant and varies with the extent of mineralization $(14,15)$ and the oestrogen status $(16)$, increases with age (17) and is different in trabecular and cortical bone (13).

Both crosslinks are released into the bloodstream after collagen break down, and are then excreted in the urine where their concentration can be measured.

Because of the different pattern of hydroxylysine glycosylation and because deoxypyridinoline is present only in bone and dentine and since both these molecules do not undergo any major liver metabolism, the urinary excretion of galactosyl hydroxylysine and deoxypyridinoline has been proposed for the estimation of the rate of bone resorption.

In recent years, some clinical studies have compared the clinical performances of these bone resorption assays $(18-20)$. The results of these studies, which are summarized in table $1 \mathrm{a}$ and $1 \mathrm{~b}$, show that the clinical performances of galactosyl hydroxylysine and deoxypyridinoline are very similar in different patient populations. Moreover, in all the clinical conditions evaluated so far, there is no indication that one marker is superior to the other. On the other hand, some data suggest that the use of the two markers together may provide better differentiation of the pathological from the normal condition $(19,20)$.

\section{Galactosyl Hydroxylysine and Deoxypyridinoline: A Methodological Comparison}

Since more and more data seem to demonstrate a high similarity in clinical performances of galactosyl hydroxylysine and deoxypyridinoline, we wanted to compare the different methodologies used to measure galactosyl hydroxylysine and deoxypyridinoline and to evaluate any differences in their practicality and reproducibility.

Both galactosyl hydroxylysine and deoxypyridinoline are measured by high performance liquid chromatography (HPLC) using fluorescence as a signal (12, 21). While deoxypyridinoline is naturally fluorescent, galactosyl hydroxylysine is not, therefore a derivatization with a fluorescent molecule is needed prior to the chromatography.

\section{Methods \\ Preanalytical procedures}

\section{Deoxypyridinoline}

The pyridinium crosslinks are present in the urine both free and in the form of peptides of different molecular mass (22). For this reason an acid hydrolysis is needed prior to the injection into the HPLC (tab. 2). Due to the very low urine concentration of pyridinoline and deoxypyridinoline (nmol/l) a preextraction is also needed.

\section{Galactosyl hydroxylysine}

Galactosyl hydroxylysine in urine is all in a free form (22b), so that no hydrolysis is needed. Furthermore the galactosyl hy-

Tab. 1a. Similarities in clinical performances between galactosyl hydroxylysine and deoxypyridinoline derived from previous publications

\begin{tabular}{|c|c|c|c|c|}
\hline & $\begin{array}{l}\text { Paget's } \\
\text { disease } \\
(\mathrm{n}=12)\end{array}$ & $\begin{array}{l}\text { Children } \\
(n=4)\end{array}$ & $\begin{array}{l}\text { Postmenopausal } \\
\text { osteoporosis } 1 \\
(n=13)\end{array}$ & $\begin{array}{l}\text { Postmenopausal } \\
\text { osteoporosis } 2 \\
(\mathrm{n}=38)\end{array}$ \\
\hline $\begin{array}{l}\text { Galactosyl hydroxylysine, Z score } \\
\text { Deoxypyridinoline, Z score } \\
\text { Galactosyl hydroxylysine, accuracy } \\
\text { Deoxypyridinoline, accuracy }\end{array}$ & $\begin{array}{l}3.43 \\
5.5 \\
90 \% \\
94 \%\end{array}$ & $\begin{array}{r}7.8 \\
14.3 \\
- \\
-\end{array}$ & $\begin{array}{l}3.34 \\
2.87 \\
81 \% \\
72 \%\end{array}$ & $\begin{array}{l}2.19 \\
2.46 \\
67 \% \pm 6 \% \\
65 \% \pm 7 \%\end{array}$ \\
\hline
\end{tabular}

Paget's disease and children were compared with normal adults $(\mathrm{n}=22)$. Osteoporosis 1 were compared ivith normal premenopausal women $(\mathrm{n}=11)$. The correlation between galactosyl hydroxylysine and deoxypyridinoline was $\mathrm{r}=0.859, \mathrm{p}<0.001$, $\mathrm{n}=51(18)$. Osteoporosis 2 were compared with postmenopausal controls $(\mathrm{n}=32)(19)$.

Tab. 1b. Similarities in clinical performances between galactosyl hydroxylysine ( $\mathrm{mmol} / \mathrm{mol}$ of creatinine) and deoxypyridinoline ( $\mu \mathrm{mol} / \mathrm{mol}$ of creatinine) derived from our unpublished data.

\begin{tabular}{lcccc}
\hline & Baseline & Low dose & Baseline & High dose \\
\hline Galactosyl hydroxylysine & $3.85 \pm 0.9$ & $4.62 \pm 1.47$ & $4.23 \pm 1.03$ & $5.49 \pm 1.89$ \\
Deoxypyridinoline & $121.2 \pm 55.7$ & $157.9 \pm 72.3$ & $100.6 \pm 26.9$ & $135.2 \pm 30.9$ \\
\hline
\end{tabular}

The excretion of the two markers was measured in patients with Ullrich-Turner syndrome before and after treatment with a low $\left(2 \mathrm{U} / \mathrm{m}^{2} \cdot \mathrm{d}\right)(\mathrm{n}=11)$ and a high $\left(3 \mathrm{U} / \mathrm{m}^{2} \cdot \mathrm{d}\right)(\mathrm{n}=9)$ dose of somatotropin (growth hormone). All the results are highly significant $\mathrm{p}<0.001$. 
Tab. 2. Deoxypyridinoline: procedures prior to injection into HPLC

- HJdrolysis:

overnight in $12 \mathrm{~mol} / 1 \mathrm{HCl}$ at $108^{\circ} \mathrm{C}$; alternatively $2-4$ hours in $12 \mathrm{~mol} / 1 \mathrm{HCl}$ at $125^{\circ} \mathrm{C}$ under pressure.

- Preextraction: on fibrous cellulose (CF1); the samples are washed with a hydrophobic buffer (1-butanol/acetic acid/water, $4+1+1$, by vol.) to separate the crosslinks. The crosslinks are then eluted with distilled water (time: 4-6h). Finally, the samples are either lyophylized or freeze-dried (time: overnight).

Total time needed for 20 samples: $36-48 \mathrm{~h}$.

Tab. 3. Galactosyl hydroxylysine: procedures prior to injection into HPLC

- Derivatization: all the samples and standards are incubated with sodium carbonate $(30 \mathrm{mmol} / \mathrm{l})$ and dansyl chloride $(10 \mathrm{~g} / \mathrm{l}$ in pure acetone) for 30 min at $60^{\circ} \mathrm{C}$. Finally, all the samples and standards are either centrifuged or filtered to remove the precipitates.

Total time needed for 20 samples: $2-3 \mathrm{~h}$.

droxylysine urinary concentration $(\mu \mathrm{mol} / \mathrm{l})$ is higher than those of pyridinoline and deoxypyridinoline, so that no preextraction is needed. On the other hand, as mentioned above, galactosyl hydroxylysine is not naturally fluorescent, and must therefore be derivatized with a fluorescent molecule. The molecule used for this purpose is dansyl chloride (tab. 3). Due to steric hindrance, glucosyl-galactosyl hydroxylysine is mainly mono-dansylated, whereas galactosyl hydroxylysine reacts with two dansyl chloride molecules $(23,23 b)$.

\section{Analytical procedures}

\section{Deoxypyridinoline}

The two pyridinium crosslinks are separated on a reverse phase column $\left(\mathrm{C}_{18}\right)$ using either a gradient (12) or an isocratic (24) chromatography.

Both the gradient and isocratic chromatography use an aqueous buffer with ammonium chloride $(0.02 \mathrm{~mol} / \mathrm{l})$, EDTA $(0.5$ $\mathrm{mmol} / \mathrm{l})$ and $n$-heptafluoro butyric acid $(0.01 \mathrm{~mol} / \mathrm{l})$ as the ionpairing agent (solvent $\mathrm{A}, \mathrm{pH} 2.0$ ) and acetonitrile as a hydrophobic agent (solvent $B$ ).

The pyridinoline and deoxypyridinoline concentrations are calculated by means of external pyridinoline and deoxypyridinoline standards, which are extracted either from bone or urine.

\section{Galactosyl hydroxylysine}

Galactosyl hydroxylysine is separated from the other molecules on a reverse phase column $\left(\mathrm{C}_{18}\right)$ using gradient.chromatography (21). The aqueous buffer is $0.05 \mathrm{~mol} / \mathrm{l}$ acetic acid, $125 \mathrm{ml} / 1$ acetonitrile and $50 \mathrm{ml} / \mathrm{l}$ propan-2-ol, $\mathrm{pH} 6.3$ (buffer $\mathrm{A}$ ) and the hydrophobic buffer is $0.05 \mathrm{~mol} / \mathrm{l}$ acetic acid, $500 \mathrm{ml} / 1$ acetonitrile and $10 \mathrm{ml} / \mathrm{l}$ propan-2-ol, pH 6.5 (buffer B).

The external standard used to calculate the galactosyl hydroxylysine concentration is $L$-lysine. $L$-Lysine was chosen (instead of $D$-lysine or hydroxylysine) because, like galactosyl hydroxy- lysine, it reacts with two molecules of dansyl chloride, therefore giving only one peak on the chromatogram, corresponding to the didansyl derivative of $L$-lysine.

The preanalytical and analytical procedures for galactosyl hydroxylysine and deoxypyridinoline are summarized and compared on table $4 a, 4 b$ and $4 c$.

Tab. 4a. Summary and comparison of galactosyl hydroxylysine and deoxypyridinoline preanalytical procedures

\begin{tabular}{lll}
\hline $\begin{array}{l}\text { Preanalytical } \\
\text { procedures }\end{array}$ & $\begin{array}{l}\text { Galactosyl } \\
\text { hydroxylysine }\end{array}$ & $\begin{array}{l}\text { Deoxy- } \\
\text { pyridinoline }\end{array}$ \\
\hline Hydrolysis & no & yes \\
Preextraction & no & yes \\
Derivatization & yes & no \\
\hline
\end{tabular}

Tab. 4b. Summary and comparison of galactosyl hydroxylysine and deoxypyridinoline analytical procedures

\begin{tabular}{|c|c|c|}
\hline $\begin{array}{l}\text { Analytical } \\
\text { procedures }\end{array}$ & $\begin{array}{l}\text { Galactosyl } \\
\text { hydroxylysine }\end{array}$ & $\begin{array}{l}\text { Deoxy- } \\
\text { pyridinoline }\end{array}$ \\
\hline System & HPLC & HPLC \\
\hline Detector & Fluorometer & Fluorometer \\
\hline Elution conditions & Gradient & $\begin{array}{l}\text { Gradient (g) or } \\
\text { isocratic (i) }\end{array}$ \\
\hline $\begin{array}{l}\text { Elution time of the } \\
\text { Compound (min) }\end{array}$ & 20 & $7(\mathrm{~g})-3(\mathrm{i})$ \\
\hline
\end{tabular}

Tab. 4c. Times required for galactosyl hydroxylysine and deoxypyridinoline analysis

\begin{tabular}{lll}
\hline & $\begin{array}{l}\text { Galactosyl } \\
\text { hydroxylysine }\end{array}$ & $\begin{array}{l}\text { Deoxy- } \\
\text { pyridinoline }\end{array}$ \\
\hline $\begin{array}{l}\text { Preanalytical procedures, } \\
\text { calculated for 20 samples (h) }\end{array}$ & $2-3$ & $36-48$ \\
$\begin{array}{l}\text { Automation } \\
\begin{array}{l}\text { Analytical procedures, } \\
\text { calculated for 1 } \\
\text { chromatogram (min) }\end{array}\end{array}$ & 40 & proposed (25) \\
Automation & yes & 30 (gradient) \\
\hline
\end{tabular}

\section{Reproducibility}

To compare the reproducibility of the two assays we collected 24-hour urine samples from two normal adults (A and B). During the 24 hours of urine collection, subject $A$ followed a diet rich in fluids while subject B followed a normal diet. Sample A was used as a low control, and sample B was considered a medium control. Galactosyl hydroxylysine was measured by the method of Moro et al. (21), while deoxypyridinoline was measured by the method of Kamel et al. with minor modifications (24). To calculate the intra-assay variation we tested samples A and B in 4-6 replicates. The test was repeated on 4 different days and the intra-assayi variation was calculated as the mean $\pm S D$ of the intra-assay coefficient of variation of each day. To calculate the inter-assay variation we tested sample $A$ and $B$ on 7 sequential days. 
Tab. 5. Galactosyl hydroxylysine and deoxypyridinoline concentration (mean \pm standard deviation) and intra-assay and interassay variation of the two measurements

\begin{tabular}{llc}
\hline & Galactosyl hydroxylysine & Deoxypyridinoline \\
\hline Intra-assay' variation & & \\
Sample A & & \\
$\mathrm{n}$ & 6 & 6 \\
Mean $\pm \mathrm{SD}$ & $1.95 \pm 0.17 \mu \mathrm{mol} / \mathrm{l}$ & $11.3 \pm 1.54 \mathrm{nmol} / \mathrm{l}$ \\
$\mathrm{CV}$ & $4.6 \pm 1.7 \%$ & $8.4 \pm 4.8 \%$ \\
Sample B & & \\
$\mathrm{n}$ & $6.07 \pm 0.35 \mu \mathrm{mol} / \mathrm{l}$ & 6 \\
Mcan $\pm \mathrm{SD}$ & $4.7 \pm 1.5 \%$ & $22.3 \pm 2.82 \mathrm{nmol} / \mathrm{l}$ \\
$\mathrm{CV}$ & & $9.2 \pm 1.8 \%$ \\
Inter-assaj' variation & & \\
Sample A & 7 & 7 \\
$\mathrm{n}$ & $1.93 \pm 0.16 \mu \mathrm{mol} / \mathrm{l}$ & $10.23 \pm 1.79 \mathrm{nmol} / 1$ \\
Mean $\pm \mathrm{SD}$ & $8.6 \%$ & $17.5 \%$ \\
$\mathrm{CV}$ & & 7 \\
Sample B & 7 & $21.6 \pm 2.49 \mathrm{nmol} / \mathrm{l}$ \\
$\mathrm{n}$ & $5.93 \pm 0.39 \mu \mathrm{mol} / \mathrm{l}$ & $11.5 \%$ \\
Mean $\pm \mathrm{SD}$ & $6.7 \%$ & \\
CV & & \\
\hline
\end{tabular}

\section{Reproducibility Results}

The results are reported in table 5. Galactosyl hydroxylysine showed a lower intra-assay variation than deoxypyridinoline with a coefficient of variation $(\mathrm{CV})$ ranging from $4.6 \pm 1.7$ (Sample A) to $4.7 \pm 1.5$ (Sample B) for galactosyl hydroxylysine and from 8.4 \pm 4.8 (Sample A) to $9.2 \pm 1.8$ (Sample B) for deoxypyridinoline. Similarly, the inter-assay variation was much lower for galactosyl hydroxylysine than for deoxypyridinoline. The galactosyl hydroxylysine inter-assay CV ranged from $8.3 \%$ (Sample A) to $6.7 \%$ (Sample B), while the deoxypyridinoline inter-assay CV ranged from $17.5 \%$ (Sample A) to $11.5 \%$ (Sample B).

\section{Discussion}

Both galactosyl hydroxylysine and deoxypyridinoline fulfil the requirements for a specific marker for bone resorption. Both the molecules are, in fact, relatively specific for bone, none of them is reutilized after the collagen breakdown and both of them seem to be excreted unchanged in the urine $(9-12)$. All the clinical studies that have compared their clinical performances have indeed shown that galactosyl hydroxylysine and deoxypyridinoline are highly correlated, have similar accuracy and discriminatory power in distinguishing subjects with altered bone resorption from normal subjects $(18-20)$. In the present study, the methods for galactosyl hydroxylysine and deoxypyridinoline determination were compared.

In addition to the similar clinical value of the two quantities, the analytical procedures for galactosyl hydroxylysine and deoxypyridinoline also show many similarities. Both molecules, for example, are measured by means of an HPLC system and detected by fluorescence. An immunoenzymatic test has also been proposed for deoxypyridinoline (22a). However, because of the high similarity between deoxypyridinoline and pyridinoline (the other pyridinium crosslink that is not bone-specific) the immunoenzymatic test responds to both of these molecules, so that only the HPLC procedure guarantees the exclusive measurement of deoxypyridinoline.

Methodologically, the methods for galactosyl hydroxylysine and deoxypyridinoline differ mainly in the preanalytical procedures. Galactosyl hydroxylysine does not need to be hydrolyzed and extracted since this molecule is present in free form in the urine (22b). On the other hand, since galactosyl hydroxylysine is not fluorescent, it must be derivatized with dansyl chloride. In contrast, deoxypyridinoline is naturally fluorescent. However, in urine, almost $50 \%$ of this molecule is in the form of peptides of different molecular mass (22). For this reason an acid hydrolysis and an extraction is needed prior to the injection into the HPLC. 
The need for a hydrolysis and a preextraction in the measurement of deoxypyridinoline implies two problems:

1) time consumption,

2) error due to the recovery.

\section{Time}

As shown in table 3c, 36-48 hours are needed for deoxypyridinoline preanalytical procedures (calculated for 20 samples) while only $2-3$ hours are needed for the galactosyl hydroxylysine derivatization (calculated for 20 samples). For this reason, the determination of deoxypyridinoline as a routine clinical procedure would require either a full-time technician or some kind of automation. An automated liquid solid extraction procedure for the pyridinium crosslinks has been recently proposed and the ability to extract 60 samples in 9 hours has been claimed by the authors (25). If confirmed, this procedure may represent a significant improvement to this important inconvenience in the pyridinium crosslinks measurement.

\section{Recovery}

The most important problem of pre-extraction, however, is the risk of error due to variable recovery of the samples. In our study both the intra-assay and inter-assay variations of deoxypyridinoline were $50-$ $100 \%$ higher than those shown for galactosyl hydroxylysine. These variations were due to the preextraction since the reproducibility for galactosyl hydroxylysine and deoxypyridinoline HPLC procedures were similar (data not shown). An appropriate internal standard is frequently used to control this important source of variation. Recently Colwell et al. (26)

\section{References}

1. Prockop, D. J., Kivirikko, K. I., Tuderman, L. \& Guzman, N. A. (1979) The biosynthesis of collagen and its disorders. N. Engl. J. Med. 301, 13-23; 77-85.

2. Prockop, D. J. \& Kivirikko, K. I. (1984) Heritable diseases of collagen. N. Engl. J. Med. 311, 376-386.

3. Nimmi, M. E. (1974) Collagen: Its structure and function in normal and pathological tissues. Sem. Arth. Reum. 4, $17-150$

4. Reid, K. B. M. (1974) A collagen-like amino acid sequence in a polypeptide chain of human $\mathrm{C1q}$ (a sub-component of the first component of complement). Biochem. J. 141, $189-208$.

5. Weiss, P. H. \& Klein, L. (1969) The quantitative relationship of urinary peptide hydroxyproline excretion to collagen degradation. J. Clin. Invest. 48, 1-10. have proposed iso-desmosine as a possible internal standard for the pyridinium crosslinks extraction, since this molecule has a different elution time on HPLC, but behaves like the pyridinium crosslinks towards the heat and the acid environment (26). Further studies are needed to confirm that the use of isodesmosine as an internal standard can decrease the important variation demonstrated so far for the deoxypyridinoline HPLC measurement.

As mentioned above, the analytical procedures for galactosyl hydroxylysine and deoxypyridinoline are similar. The HPLC analysis of deoxypyridinoline is, however, more rapid (because of the preextraction), and isocratic elution conditions can be used. Moreover, the preextraction allows the injection of a purer sample into the HPLC, and more runs can therefore be made on the same column for the deoxypyridinoline measurement. The analytical procedures of both galactosyl hydroxylysine and deoxypyridinoline can be easily automated by using an HPLC system with an autosampler and a computer.

In conclusion we have reviewed our knowledge of galactosyl hydroxylysine and deoxypyridinoline with particular emphasis on the practicality and the reproducibility of their measurements, and we have shown that the major differences between galactosyl hydroxylysine and deoxypyridinoline assays are the preanalytical hydrolysis and an extraction, which must be performed before the deoxypyridinoline assay. The deoxypyridinoline measurement consequently requires longer time for its operation, and its recovery is more variable.

\section{Acknowledgement}

This research was supported by the Italian Ministry of the University and Scientific Technol. Res.-MURST.
6. Efron, M. L., Bixby, E. M. \& Pryles, C. V. (1969) Hydroxyprolinemia. II. A rare metabolic disease due to deficiency of the enzyme "hydroxyproline oxidase". N. Engl. J. Med. 272, 1299-1309.

7. Horlein, D., Fietzek, P. P. \& Kuhn, K. (1978) Pro-gln: The procollagen peptidase cleavage site in the a1(I) chain of dermatosparatic calf skin procollagen. FEBS Lett. 13, $279-282$.

8. Gallagher, J. A., Guenther, H. L. \& Fleisch, H. A. (1982) Rapid intracellular degradation of newly synthetized collagen by bone cells. Effect of.dichloromethylenebiphosphonate. Biochim. Biophys. Acta 719, 349-355.

9. Pinnell, S. R., Rox, R. \& Krane, S. M. (1971) Human collagens: differences in glycosylated hydroxylysine in skin and bone. Biochim. Biophys. Acta 239, 119-122. 
10. Krane, S. M., Kantrowitz, F. G., Byrne, M., Pinnell, S. R. \& Singer, F. R. (1977) Urinary excretion of hydroxylysine and its glycosides as an index of collagen degradation. J. Clin. Invest. 59, 819-827.

10b. Sternberg, M. \& Spiro, R. G. (1980) Studies on the catabolism of the hydroxylysine-linked disaccharide units of basement membranes and collagens. Isolation and characterization of a rat kidney $\alpha$-glucosidase of high specificity. J. Biol. Chem. 254, 10329-10336.

10c. Moro, L., Noris-Suarez, N., Michalsky, M., Romanello, M. \& de Bernard, B. (1992) The glycosides of hydroxylysine are final products of collagen degradation in humans. Biochim. Biophys. Acta, accepted in press.

11. Segrest, J. P. \& Cunningham, I. W. (1979) Variations in human urinary O-hydroxylysyl glycoside levels and their relationship to collagen metabolism. J. Clin. Invest. 49, 1497-1509.

11b. Robins, S. P. (1980) Turnover of collagen and its precursor. In: Biology of Collagen (Viidick, A. \& Wust, J., eds.) Acad. Press, N. Y. p. 135.

12. Eyre, D. R., Koob, T. J. \& Van Ness, K. P. (1984) Quantification of hydroxypyridinium crosslinks in collagen by high-performance liquid chromatography. Analyt. Biochem. 137, 380-388.

13. Robins, S. P. \& Duncan, A. (1987) Pyridinium crosslinks of bone collagen and their location in peptides isolated from rat femur. Biochim. Biophys. Acta 914, 233-239.

14. Linde, A. \& Robins, S. P. (1988) Quantitative assessment of collagen crosslin ks in dissected predentine and dentine. Collagen Rel. Res. 8, 443-450.

15. Shikata, H., Utsumi, N., Hiramatsu, M., Noguchi, M. \& Fujimoto, D. (1986) Effect of calcium deficiency on the content of nonreducible crosslinks in rat mandibular bone. Metabolism 35, 206-208.

16. Shimizu, K., Higuchi, K., Yamamuro, T., Ohtsuji, T. \& Takeda, T. (1982) Increase in pyridinoline cross-linking of mouse collagen induced by estrogen. Experientia 38 , 864-887.

17. Shikata, H., Hiramatsu, M., Masumizu, T., Fujimoto, D. \& Utsumi, N. (1985) Age-related changes in the content of non-reducible crosslinks in rat mandibular bone. Archs. Oral Biol. 30, $451-453$.

18. Bettica, P., Moro, L., Robins, S., Taylor, A. K., Talbot, J., Singer, F. \& Baylink, D. J. (1992) Bone resorption markers galactosyl hydroxylysine, pyridinium crosslinks and hydroxyproline compared. Clin. Chem. 38, 23132318.

19. Bettica, P., Taylor, A. K., Talbot, J., Moro, L. \& Baylink, D. J. (1992) Accuracy and discriminate power of galactosyl hydroxylysine and deoxypyridinoline in postmenopausal osteoporosis. Accepted for oral presentation at the XXIII European Symposium on Calcified Tissues, Heidelberg, April 25-29 1993.
20. Bettica, P., Moro, L., Robins, S. P., Taylor, A. K., Morales-Piga, A., Talbot, J. \& Baylink, D. J. (1992) Urine galactosyl hydroxylysine and pyridinoline in parallel distinguish osteoporotic patients from controls. Connective Tissue Res. 27, 187.

21. Moro, L., Gazzarrini, C., Modricky, C., Rovis, L., de Bernard, B., Galligoni, E., Crovellari, D., Morassut, S. \& Monfardini, S. (1990) High predictivity of galactosyl hydroxylysine in urine as an indicator of bone metastases from breast cancer. Clin. Chem. 36, 772-774.

22. Daniloff, Y., Harris, D., Shepard, K. \& Seyedin, S. (1992) Appearance of hydroxypyridine crosslinks in urinc. Connective Tissue Res. 27, 187.

22a. Robins, S., Stewart, P., Astbury, C. \& Howard, A. B. (1986) Measurement of the cross linking compound, pyridinoline, in urine as an index of collagen degradation in joint diseasc. Ann. Rheum. Dis. 45, 969-973.

22b. Askenasi, R. (1973) A new rapid method for measuring hydroxylysine and its glycosides in hydrolysates and physiological fluids. Biochim. Biophys. Acta 304, 375-383.

23. Moro, L., Modricky, C., Stagni, N., Vittur, F. \& de Bernard, B. (1984) High-performance liquid chromatographic analysis of urinary hydroxylysyl glycosides as indicators of collagen turnover. Analyst 109, 1621-1622.

23b. Moro, L., Battista, C., Modricky, C., Rovis, L. \& de Bernard, B. (1989) High performance liquid chromatographic preparation of galactosyl-hydroxylysine, a specific bone collagen marker. J. Chromatogr. 490, 285- 292.

24. Kamel, S., Brazier, M., Desmet, G., Picard, C., Mennecier, I. \& Sebert, J. I. (1992) High-performance liquid chromatographic determination of 3-hydroxypyridinium derivatives as new markers of bone resorption. J. Chromatography $574,255-260$.

25. Abbiati, G., Rigoldi, M., Frignani, S., Arrigoni, M. \& Castiglioni, C. (1992) Determination of pyridinoline (HP) and deoxypyridinoline (LP) in urine by automated liquid solid extraction procedure in combination with HPLC. Bone Miner. 17 (Suppl. 1) 129.

26. Colwell, A., Eastell, R. \& Russell, R. G. G. (1992) Evaluation of internal standards for use in high performance liquid chromatographic assay of urinary hydroxypyridinium compounds. J. Bone Miner. Res. 7 (Suppl. 1) S 268.

Pror. Luigi Moro

Dipartimento di Biochimica

Biofisica e Chimica delle Macromolecole Universita' degli Studi di Trieste

Via L. Giorgeri 1

I-34127-Trieste

Italy 
